\title{
Effects of a blend of Saccharomyces cerevisiae-based direct-fed microbial and fermentation products on plasma carbonyl- metabolome and fecal bacterial community of beef steers
}

James A. Adeyemi ${ }^{1}$, Sunday O. Peters ${ }^{2}$, Marcos De Donato ${ }^{3}$, Andres Pech Cervantes $^{4}$ and Ibukun M. Ogunade ${ }^{1 *}$ (D)

\begin{abstract}
Background: Previous studies have evaluated the metabolic status of animals fed direct-fed microbial (DFM) using enzyme-based assays which are time-consuming and limited to a few metabolites. In addition, little emphasis has been placed on investigating the effects of DFM on hindgut microbiota. We examined the effects of dietary supplementation of a blend of Saccharomyces cerevisiae-based DFM and fermentation products on the plasma concentrations of carbonyl-containing metabolites via a metabolomics approach, and fecal bacterial community, via 165 rRNA gene sequencing, of beef steers during a 42-day receiving period. Forty newly weaned steers were randomly assigned to receive a basal diet with no additive $(\mathrm{CON} ; n=20)$ or a basal diet supplemented with $19 \mathrm{~g}$ of Commence $^{\text {TM }}($ PROB; $n=20)$ for a 42-day period. Commence ${ }^{\mathrm{TM}}\left(\mathrm{PMl}\right.$, Arden Hills, MN) is a blend of $6.2 \times 10^{11} \mathrm{cfu} / \mathrm{g}$ of S. cerevisiae, $3.5 \times 10^{10} \mathrm{cfu} / \mathrm{g}$ of a mixture of Enterococcus lactis, Bacillus subtilis, Enterococcus faecium, and Lactobacillus casei, and the fermentation products of these aforementioned microorganisms and those of Aspergillus oryzae and Aspergillus niger. On d 0 and 40, rectal fecal samples were collected randomly from 10 steers from each treatment group. On d 42, blood was collected for plasma preparation.
\end{abstract}

Results: A total number of 812 plasma metabolites were detected. Up to 305 metabolites [fold change (FC) $\geq 1.5$, FDR $\leq 0.01$ including glucose, hippuric acid, and 5-hydroxykynurenamine were increased by PROB supplementation, whereas 199 metabolites ( $F C \leq 0.63, F D R \leq 0.01)$ including acetoacetate were reduced. Supplementation of PROB increased $(P \leq 0.05)$ the relative abundance of Prevotellaceae UCG-003, Megasphaera, Dorea, Acetitomaculum, and Blautia. In contrast, the relative abundance of Elusimicrobium, Moheibacter, Stenotrophomonas, Comamonas, and uncultured bacterium belonging to family p-2534-18B5 gut group (phylum Bacteroidetes) were reduced ( $P \leq 0.05)$.

Conclusions: The results of this study demonstrated that supplementation of PROB altered both the plasma carbonyl metabolome towards increased glucose concentration suggesting an improved energy status, and fecal bacterial community, suggesting an increased hindgut fermentation of the beef steers.

Keywords: Beef steer, Carbonyl, Fecal bacteria, Lactate-utilizing bacteria, Plasma metabolomics

\footnotetext{
* Correspondence: ibukun.ogunade@kysu.edu

${ }^{1}$ College of Agriculture, Communities, and the Environment, Kentucky State

University, Frankfort, KY 40601, USA

Full list of author information is available at the end of the article
}

(c) The Author(s). 2020 Open Access This article is distributed under the terms of the Creative Commons Attribution 4.0 International License (http://creativecommons.org/licenses/by/4.0/) which permits unrestricted use, distribution, and reproduction in any medium, provided you give appropriate credit to the original author(s) and the source, provide a link to the Creative Commons license, and indicate if changes were made. The Creative Commons Public Domain Dedication waiver (http://creativecommons.org/publicdomain/zero/1.0/) applies to the data made available in this article, unless otherwise stated. 


\section{Background}

Direct-fed microbials (DFM) are commonly used in livestock production systems to improve the metabolic and energy status of animals especially during stress periods, thereby leading to improved animal productivity [1-3]. Several studies have suggested that the effects of DFM on the metabolic and energy status of ruminants are attributed to the modulation of the rumen microbiota, improved gut integrity, and increased intestinal nutrient absorption [4-6]. The majority of studies using DFM have been targeted toward enhancing metabolism of lactic acid to propionate through inoculation of Saccharomyces cerevisiae and lactate-utilizing bacteria [1, 2]. Other studies have evaluated the approach of using lactic acid-producing bacteria, such as Lactobacillus and Enterococcus spp., to enhance production of lactic acid which can be metabolized to propionate by ruminal lactate-utilizing bacteria [3]. In recent years, most commercial DFM products are formulated to contain a mixture of these aforementioned micro-organisms and their fermentation products in order to ensure efficacies and multi-factorial response.

Previous studies have evaluated the metabolic status of animals fed supplemental DFM using enzyme-based assays for individually determining certain metabolites, such as blood glucose and beta-hydroxybutyric acid [3, 7]; however, these assays are time-consuming and limited to a few metabolites. Recent advances in metabolomics have provided the opportunity to simultaneously quantify the relative concentrations of multiple metabolites [8]. Metabolites containing a carbonyl group are important classes of molecules including ketones and aldehydes, such as steroids and sugars [9]. These metabolites are common intermediates of energy metabolism; therefore, their concentrations in the blood can reflect the energy status of animals. The use of highperformance chemical isotope labeling (CIL) liquid chromatography mass spectrometry (LC-MS)-based metabolomics has provided the opportunity to analyze the carbonyl submetabolome of biofluid with high coverage, accuracy, and precision [9]. Analysis of plasma carbonylmetabolome of animals will provide an opportunity to understand better how dietary supplementation of DFM affects animal performance and health.

In our companion study [10], supplementation of a blend of $S$. cerevisiae-based DFM and fermentation products (PROB) improved the performance and health of newly-weaned beef steers which might be attributed to improved energy status of the animals. Therefore, we hypothesized that supplementation of PROB would alter the plasma carbonyl submetabolome due to its positive effects on the performance of the steers. The objective of this study was to apply a CIL/LC-MS-based quantitative untargeted metabolomics to evaluate the effects of
PROB on the plasma concentrations of carbonylcontaining metabolites in beef steer.

Also in our companion study [10], there was a tendency for a lower fecal $\mathrm{pH}$ in steers fed PROB diet, which is possibly an indication of increased volatile fatty acids due to increased hindgut fermentation and altered hindgut microbiota. Little emphasis has been placed on investigating the effects of DFM on hindgut microbiota. The fecal $\mathrm{pH}$ and bacterial composition can particularly reflect the true condition of the hindgut microbiota [11]. Therefore, the second objective of this study was to evaluate the effects of PROB supplementation on fecal bacterial community of beef steers.

\section{Methods}

The Kentucky State University Animal Care and Use Committee approved all procedures and protocols for this experiment.

\section{Animals and feeding}

Forty newly-weaned Angus crossbred steer calves [7 d post-weaning; $210 \pm 12 \mathrm{~kg}$ of body weight (BW); $180 \pm 17$ $\mathrm{d}$ of age] were stratified by BW into 4 weight blocks. The steers were randomly assigned (within each weight block) to 1 of 2 treatments and housed in individual slatted floor pens ( 1 steer per pen). These calves were from a single source and were not transported. The treatments were a basal diet with no additive (CON; $n=20)$ or a basal diet supplemented with $19 \mathrm{~g} / \mathrm{d}$ of Commence ${ }^{\mathrm{Tm}}$ (PROB; $n=20)$ for a 42-day period. Commence ${ }^{\mathrm{Tm}}(\mathrm{PMI}$, Arden Hills, MN) contains a blend of $6.2 \times 10^{11} \mathrm{cfu} / \mathrm{g}$ of S. cerevisiae, $3.5 \times 10^{10} \mathrm{cfu} / \mathrm{g}$ of a mixture of Enterococcus lactis, Bacillus subtilis, Enterococcus faecium, and Lactobacillus casei, and the fermentation products of these aforementioned microorganisms as well as those of $A s$ pergillus oryzae and Aspergillus niger. The basal diet was fed daily as a total mixed ration (TMR) at 08:00 h (See Additional file 1: Table S1). The additive was topdressed daily on the TMR in the form of a premix using dried distillers grains with solubles for the PROB treatment while a similar premix with no additive was topdressed for the CON treatment.

\section{Feces and blood collection}

On d 0 and 40, rectal fecal samples were collected randomly from 20 steers (10 steers from each treatment) approximately $4 \mathrm{~h}$ after feeding and immediately stored at $-80^{\circ} \mathrm{C}$ until further analysis. On d $42,15 \mathrm{~mL}$ of blood was taken before the morning feeding. The blood was collected from the coccygeal vessels into tubes containing sodium heparin (ThermoFisher Scientific, Wilmington, DE) for preparation of plasma and immediately stored at $-80^{\circ} \mathrm{C}$ until further analysis. 


\section{Analysis of carbonyl-containing metabolites}

Relative quantification of metabolites containing a carbonyl group (carbonyl-metabolome) in plasma samples collected on d 42 was done using a CIL/LC-MS method. This method uses a differential ${ }^{12} \mathrm{C}$ - and ${ }^{13} \mathrm{C}$ - dansylhydrazine $(\mathrm{DnsHz})$ labeling to change the chemical and physical properties of carbonyl metabolites to enable them to be efficiently separated by LC and ionized by electrospray ionization MS [9]. The workflow of the analysis as well as details of dansylhydrazine labeling, LCUV normalization, and LC-QTOF-MS setup and running conditions have been previously reported [9]. Briefly, $90 \mu \mathrm{L}$ of LC-MS grade methanol was mixed with $30 \mu \mathrm{L}$ of plasma sample for protein precipitation. The methanol extract was then dried after incubation at $-20^{\circ} \mathrm{C}$ for $2 \mathrm{~h}$ and then re-dissolved in $30 \mu \mathrm{L}$ of LC-MS grade water. For labeling, $30 \mu \mathrm{L}$ of $\mathrm{pH}$-adjusting reagent and $30 \mu \mathrm{L}$ of ${ }^{12} \mathrm{C}_{2}$-labeling was mixed with $20 \mu \mathrm{L}$ of the individual plasma samples and the pooled sample (prepared by mixing $70 \mu \mathrm{L}$ of each plasma sample). In addition, $30 \mu \mathrm{L}$ of $\mathrm{pH}$ adjusting reagent and $30 \mu \mathrm{L}$ of ${ }^{13} \mathrm{C}_{2}$-labeling was mixed with another $20 \mu \mathrm{L}$ of pooled sample. After vortexing and spinning down (using a SpeedVac to remove the acid catalyst), the mixture was incubated at $40^{\circ} \mathrm{C}$ for $60 \mathrm{~min}$. Then the mixture was cooled down in $-80^{\circ} \mathrm{C}$ freezer for $10 \mathrm{~min}$ to stop the labeling reaction. The mixture was then dried using a centrifugal vacuum concentrator. Finally, the dried mixture containing the labelled metabolites was redissolved in $100 \mu \mathrm{L}$ of acetonitrile/Water $(50: 50, \mathrm{v} / \mathrm{v})$. The ${ }^{12} \mathrm{C}_{2}$-labeled individual sample was mixed with ${ }^{13} \mathrm{C}_{2}$-labeled reference sample in equal amount according to carbonyl-labeling LC-UV normalization results. The solution was diluted and centrifuged at $15294 \times g$ for $10 \mathrm{~min}$ before injecting into Bruker Elute LC linked to Bruker Impact II QTOF MS for analysis. Quality control (QC) sample (prepared by mixing equal amount of a ${ }^{12} \mathrm{C}$-labeled and a ${ }^{13} \mathrm{C}$-labeled pooled sample) was injected every 10 sample runs to monitor instrument performance.

\section{Fecal DNA extraction, sequencing, and diversity analysis}

Total DNA was extracted from the feces with a PowerSoil DNA Isolation Kit (MOBIO Laboratories Inc.; Carlsbad, CA, USA). The integrity of the DNA was verified using $0.8 \%$ agarose gel electrophoresis. DNA concentration was determined using a NanoDrop 3300 (ThermoFisher Scientific, Wilmington, DE). The DNA samples were prepared for sequencing according to the Illumina 16S Metagenomic Sequencing Library protocols to amplify the V3-V4 region. Amplification of the genomic DNA (10 ng) was performed using polymerase chain reaction (PCR) conditions: $94{ }^{\circ} \mathrm{C}$ for $3 \mathrm{~min}$ followed by 35 cycles of $94^{\circ} \mathrm{C}$ for $15 \mathrm{~s}, 55^{\circ} \mathrm{C}$ for $45 \mathrm{~s}$, and $72{ }^{\circ} \mathrm{C}$ for $1 \mathrm{~min}$, followed by a final elongation step of 8 min at $72^{\circ} \mathrm{C}$. 519F:5'-CCTACGGGNGGCWGCAG-3' and 806R: 5'-GACTACHVGGGTATCTAATCC-3' were used as the primer sequences. Quantification of the final purified product was done according to qPCR Quantification Protocol Guide (KAPA Library Quantification kits for Illumina Sequencing platforms, Wilminton, MA, USA), and the quality was checked using the LabChip GX HT DNA High Sensitivity Kit (PerkinElmer; Waltham, MA, USA). Sequencing was performed on a paired-end Illumina MiSeq platform to generate 300-bp paired-end raw reads. Raw paired end reads were joined using FLASH2 v.c41a82e [12]. The merged reads were quality-filtered to remove adaptors and low quality bases using filtering conditions of Trimmomatic v0.33 [13]. The resulting trimmed reads were compared with the reference database (the "Gold" database, [14]) using the UCHIME algorithm [15] to detect and remove the chimeric sequences. After processing and quality filtering, UCLUST in QIIME (version 1.8.0 [15];) was used to cluster the tags with $97 \%$ similarity to acquire the Operational Taxonomic Units (OTU). The representative OTU sequences were annotated using the rRNA database (Silva) for taxonomic assignment. Shannon index, a measure of alpha diversity, and unweighted unifrac distance, a measure of beta diversity were generated using the QIIME software package with a script core_diversity_analyses.py [16]. The datasets in this study are available in the NCBI BioProject database with the SRA accession number: PRJNA588450.

\section{Data and statistical analysis}

Variables such as relative abundance of fecal bacteria and diversity indices were analyzed as a randomized complete block design using the GLIMMIX procedure of SAS 9.4 (SAS Inst. Inc., Cary, NC). The model included the fixed effects of treatment, random effects of block (BW as the block). Day 0 data were used as covariates. Each steer housed in an individual pen served as the experimental unit. Linear discriminant analysis effect size (LEfSe), which uses a Kruskal-Wallis (KW) test and the logarithmic linear discriminant analysis (LDA) score, was used to identify the most differentially abundant taxa [17]. The significance estimate for the KW test and the LDA score cutoff were 0.05 and 4.0, respectively.

For the metabolomics data, 48 LC-MS data (4 blank group samples, 4 QC samples, $20 \mathrm{CON}$ samples, and 20 PROB samples) were first exported to .csv file with Bruker DataAnalysis 4.4. The exported data were uploaded to IsoMS Pro 1.0.10. After format conversion, data quality check and data processing were performed according to procedures described by Mung and Li [18]. Metabolite identification was performed using a three-tier identification approach. In tier 1, peak pairs were searched against the CIL Library [DnsHz (ketone and aldehyde)] 
based on accurate mass and retention time [19]. In tier 2 , high-confidence identification based on accurate mass and predicted retention time matches was performed using linked identity library (LI Library) [20]. In tier 3, the remaining peak pairs were searched, based on accurate mass match, against the MyCompoundID (MCID) library composed of 8,021 known human endogenous metabolites (zero-reaction library) and their predicted metabolic products from one metabolic reaction (onereaction library) and two metabolic reactions (two-reaction library) [20]. The mass accuracy tolerance window was set at $10 \mathrm{ppm}$ for all searches, and the retention time tolerance window was set to $30 \mathrm{~s}$ for CIL library and $200 \mathrm{~s}$ for LI library.

Partial least squares discriminant analysis (PLS-DA) scores plot was used to visually show the differences between plasma metabolome of CON and PROB. Univariate (volcano plot) was generated using IsoMS Pro 1.0 [18] by plotting the fold change (FC; PROB/CON) against the false discovery rate (FDR) adjusted $P$-value. Relative concentrations of metabolites with $\mathrm{FC} \geq 1.5$ or $\leq$ 0.67 having FDR $\leq 0.01$ were differentially increased or decreased relative to CON, respectively. Pearson correlation analysis was used to examine the association between the relative concentrations of the identified plasma metabolites and growth performance indices (feed efficiency (FE) and average daily gain (ADG). The Pearson correlation coefficients were generated using $R$ software (http://www.r-project.org). Pearson correlations were declared significant at $P \leq 0.10$.

\section{Results}

The effects of PROB supplementation on performance and health of the beef steers were reported in our previous study [10]. Briefly, compared with CON, dietary supplementation of PROB increased final body weight (270 vs. $260 \mathrm{~kg} ; P=0.01 ; \mathrm{SE}=2.67)$ and ADG (1.42 vs. $1.23 \mathrm{~kg} ; P=$ 0.04; $\mathrm{SE}=0.06)$. There was a tendency for improved $\mathrm{FE}$ with PROB supplementation $(0.232$ vs $0.209 ; P=0.10$; $\mathrm{SE}=0.01)$. There was a tendency for lower fecal $\mathrm{pH}(P=$ $0.08 ; 6.46$ vs. 6.62 ; $\mathrm{SE}=0.06)$ in steers fed $\mathrm{PROB}$ diet.

\section{Effects of PROB on plasma carbonyl-containing metabolites}

The mass-to-charge ratio $(\mathrm{m} / \mathrm{z})$ of 251.0849 was used as a background peak to check the mass accuracy of all the samples as well as 4 QC and 4 blank group samples. The appearance and mass of the peak was consistent for all runs and all scanned $\mathrm{m} / \mathrm{z}$ were within the expected range, showing good stability and mass accuracy for the data acquisition (See Additional file 2: Figure S1). Approximately $757 \pm 44$ peak pairs per run were detected (See Additional file 3: Table S2) and a total number of 812 unique peak pairs were detected (See Additional file 4).
Fourteen peak pairs were positively identified in tier 1, 29 peak pairs were putatively identified with high confidence in tier 2 while 104, 434 and 661 peak pairs were matched in the zero-, one- and two-reaction libraries, respectively in tier 3. Thus, 704 pairs (86.7\% of 812 unique pairs) were positively identified or putatively matched. Both PCA and PLS-DA plots showed clear separations between the CON and PROB samples, indicating that the plasma carbonylcontaining metabolome of the beef steers was altered by PROB supplementation (Fig. 1). The permutation test result $(P=0.01)$ and PLS-DA cross validation results $\left(R^{2}=\right.$ $\left.0.988, Q^{2}=0.788\right)$ confirm the validity of the PLS-DA model (See Additional file 5: Figure S2).

Volcano plot analysis showed that 305 metabolites ( $F C \geq 1.5, F D R \leq 0.01$, in red) were increased by $P R O B$ supplementation, whereas 199 metabolites $(\mathrm{FC} \leq 0.63$, FDR $\leq 0.01$, in blue) were reduced (Fig. 2). Among those that were differentially altered, 9 metabolites were positively identified in tier 1, 17 metabolites were putatively identified with high confidence in tier 2, and 413 metabolites were matched in tier 3 (See Additional file 6). The differentially altered ( $\mathrm{FC} \geq 1.5$ or $\leq 0.67$, FDR $\leq 0.01$ ) metabolites that were positively and putatively identified with high confidence are shown in Table 1. Relative to $\mathrm{CON}, 23$ metabolites including glucose, fructose, galactose, glyceraldehyde, hippuric acid, glycoaldehyde, and 5hydroxykynurenamine were increased (FC $\geq 1.5$, FDR $\leq$ 0.01 ) in steers fed PROB diet. In contrast, 3 metabolites (lactose, acetoacetate, and 2-dehydro-3-deoxy-L-arabinonate) were decreased $(\mathrm{FC} \leq 0.63, \mathrm{FDR} \leq 0.01)$.

Plasma concentrations of 5-oxopentanoate, isomer of (S)-2-aceto-2-hydroxybutanoate, and isomer of (S)-3methyl-2-oxopentanoic acid were positively correlated with both FE and ADG while relative concentrations of 3-(4-hydroxyphenyl)pyruvate, (S)-2-aceto-2-hydroxybutanoate, (R)-3-hydroxy-3-methyl-2-oxopentanoate, and 2-dehydropantoate were positively correlated with ADG (Table 2).

\section{Effects of PROB on fecal bacterial community}

After quality filtering and the removal of chimeric sequences, a total number of $1,408,962$ sequences were produced (an average of 70,448 sequences per sample). The mean read length of quality checked and merged reads was $451 \mathrm{bp}$.

There were no treatment effects $(P>0.10)$ on alpha (Shannon index) and beta diversity (unweighted unifrac distance) (See Additional file 7: Figure S3). Supplementation of PROB increased $(P \leq 0.05)$ the relative abundance of Prevotellaceae UCG-003, Megasphaera, Dorea, Acetitomaculum, and Blautia. In contrast, the relative abundance of Elusimicrobium, Moheibacter, Stenotrophomonas, Comamonas, and uncultured bacterium belonging to family p-2534-18B5 gut group were reduced $(P \leq 0.05)$ (Table 3$)$. Using LEfSe, 


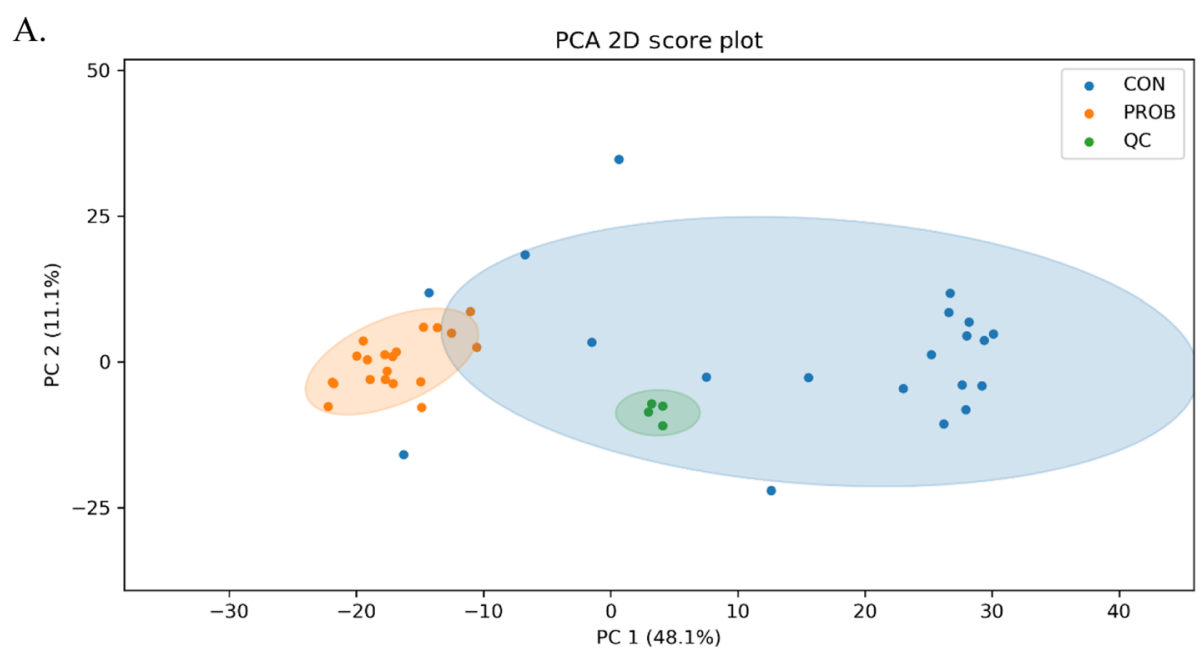

B.

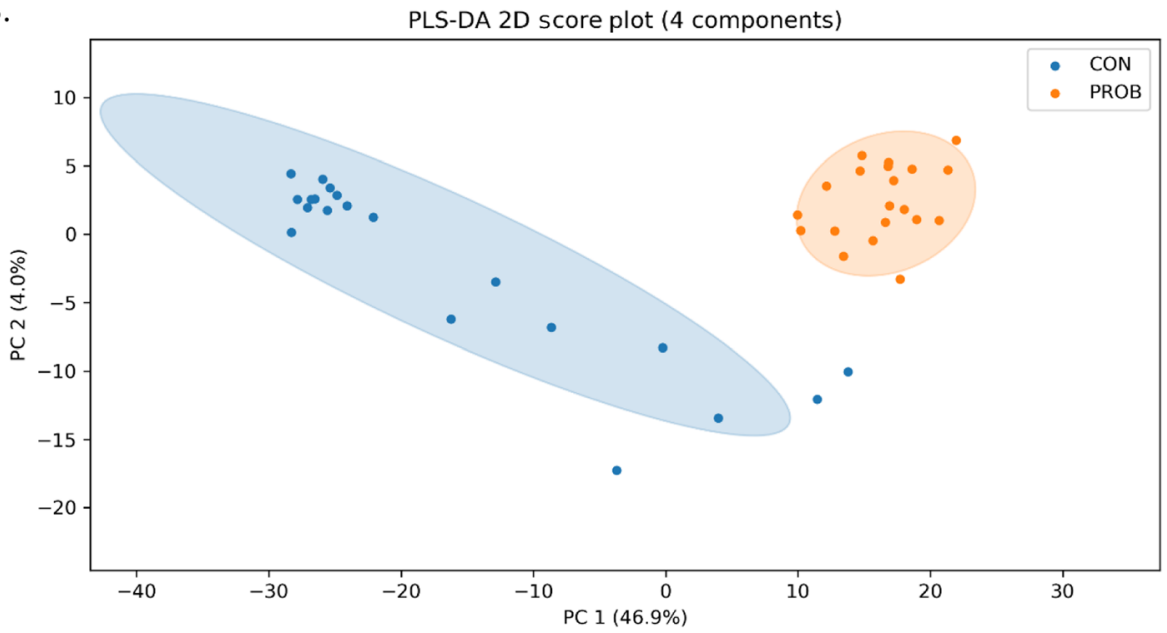

Fig. 1 a Principle component analysis (PCA) scores plot (with quality control), b Partial least squares discriminant analysis (PLS-DA) scores plot of the two treatments. $\mathrm{CON}=$ control; $\mathrm{PROB}=\mathrm{a}$ blend of Saccharomyces cerevisiae-based direct-fed microbial and fermentation products fed at $19 \mathrm{~g} / \mathrm{steer}$ per day

Prevotellaceae UCG-003 was the only altered taxon with LDA score $=4.28$ at the genus level (Fig. 3).

\section{Discussion}

\section{Effects of PROB on plasma carbonyl-metabolome}

The increased relative concentrations of plasma monosaccharides such as glucose, galactose, fructose, and glyceraldehyde in steers fed PROB diet is evidence of an improved energy status of the animals. Glucose is the primary energy source and must be sufficient in the blood stream for optimum performance of animals [21, 22]. In ruminants, glucose entry into the systemic circulation is majorly via hepatic or renal gluconeogenesis because glucose supply from direct intestinal absorption is low [22]. The major precursors for glucose synthesis in ruminants are short chain fatty acids (propionate, valerate, isobutyrate), lactate, and amino acids [21, 22]. Of all the precursors, propionate is the predominant substrate for gluconeogenesis in ruminants [22, 23]. The additive used in this study contains several microorganisms including S. cerevisiae and lactic acid bacteria (LAB) such as Enterococcus lactis, Enterococcus faecium, and Lactobacillus casei. Saccharomyces cerevisiae favors the activities of lactic acid utilizing bacteria (such as Selenomonas ruminantium and Megasphaera elsdenii) in the rumen [24, 25]. Production of lactic acid in the rumen by LAB is accompanied by the growth of lactic acid utilizing bacteria, which can metabolize lactate directly to propionate or indirectly through succinate to propionate $[23,26]$. Therefore, it is reasonable to speculate that PROB supplementation altered rumen fermentation acid profile towards increased production of 


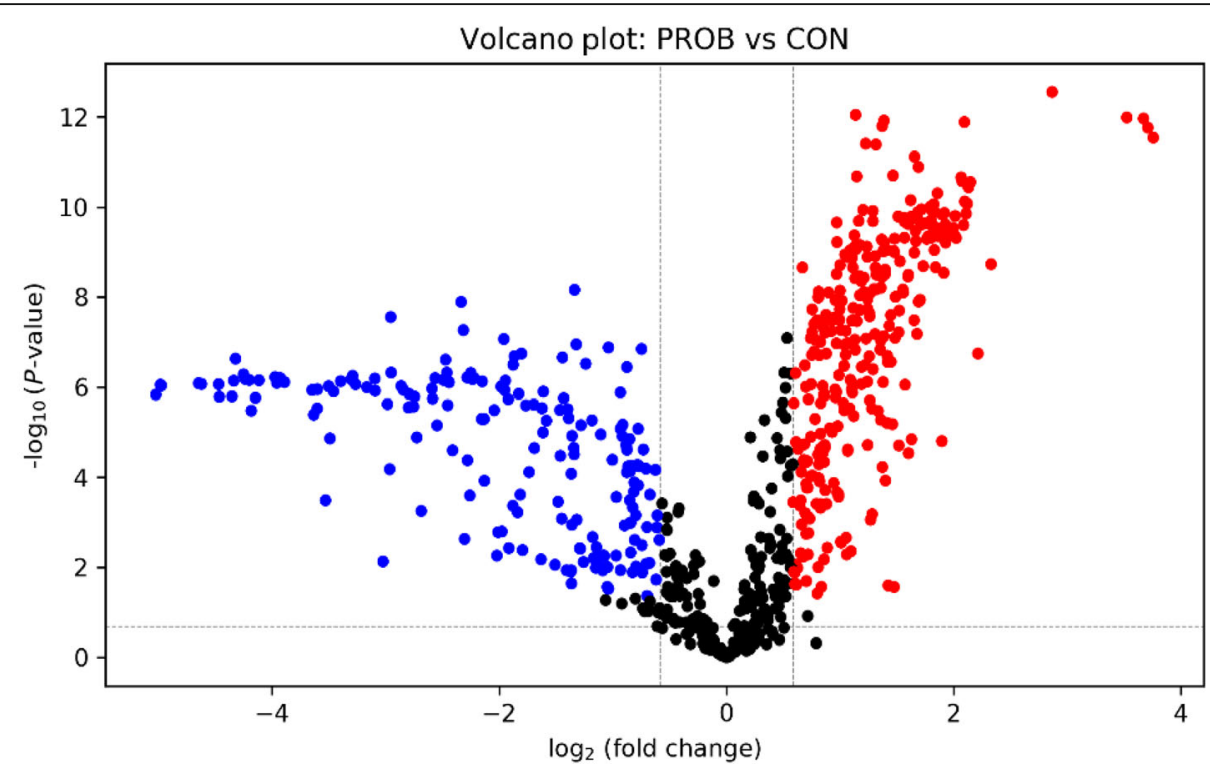

Fig. 2 Volcano plot showing the differential metabolites. FC $>1.5, F D R \leq 0.05$ (in red): significantly increased relative to $C O N$. FC $<0.67$, FDR $\leq 0.05$ (in blue): significantly reduced relative to $\mathrm{CON}$. $\mathrm{CON}=$ control; $\mathrm{PROB}=$ a blend of Saccharomyces cerevisiae-based direct-fed microbial and fermentation products fed at $19 \mathrm{~g} / \mathrm{steer}$ per day

propionate, thereby resulting to increased glucose supply. Predictably, increased plasma concentration of glucose was accompanied by decreased plasma acetoacetate concentration. Acetoacetate is one of the three major ketone bodies (others are acetone and beta-hydroxybutyric acid) produced in the liver cells from the break down of fatty acids as a source of energy when carbohydrates are in short supply [27]. Nocek et al. [28] investigated the effect of supplementing DFM containing a combination of 2 Enterococcus strains $\left(5 \times 10^{9} \mathrm{cfu} / \mathrm{g}\right)$ and $S$. cerevisiae $\left(2 \times 10^{9} \mathrm{cfu} / \mathrm{g}\right)$ to lactating Holstein cows during the transition period and reported improved performance associated with higher blood levels of glucose and lower serum levels of beta-hydroxybutyric acid. Similar results were reported by Nocek and Kautz [29].

Compared to $\mathrm{CON}$, greater plasma concentration of hippuric acid was observed in steers fed PROB diet. Hippuric acid is a gut-derived phenolic metabolite formed by the conjugation of benzoic acid (produced by microbial degradation of phenolic compounds) with glycine in the liver or kidney [30]. Studies in rats and humans have demonstrated that urinary and blood hippuric acid content is modulated by changes in the intestinal microbiome [31, 32]. Although rumen microbial population was not measured in the current study, increased plasma hippuric acid concentration may possibly be because PROB supplementation modulates the composition of rumen microbiota towards a more favorable production of hippuric acid. In humans, increased fasting serum hippuric acid concentration was associated with better glucose and insulin metabolism [33]. In addition, $\mathrm{Wu}$ et al. [34] reported increased serum hippuric acid concentration in highly efficient dairy cows. Consequently, it is reasonable to speculate that higher plasma hippuric acid in steers fed PROB diet indicates improved glucose metabolism, which corroborates increased plasma glucose concentration observed in animals fed PROB diet. Further studies are needed to infer the connection between plasma hippuric acid and energy status of animals.

Recent studies have identified tryptophan catabolism, through the kynurenine metabolic pathway, as one of the mechanisms by which the immune system modulates the balance between response to pathogens and tolerance to harmless antigens [35]. The enzyme, indoleamine 2, 3-dioxygenase that breaks down tryptophan via this pathway is induced by the immune cells during activation. Increased plasma concentration of 5hydroxykynurenamine, an intermediate product of tryptophan catabolism [36], suggests an increased tryptophan degradation possibly due to immune cell activation. This is in line with the results of our companion study [10], which showed that PROB supplementation increased the expression of immune-related genes responsible for regulating the animal's immune response toward intracellular and extracellular pathogens.

Phenylacetylglycine levels in the blood is primarily driven by gut microbial metabolism [37]. Formation of phenylacetylglycine by conjugation of phenylacetate has been reported to be an ammonia-lowering pathway [38]. Although previous studies have reported decreased ruminal ammonia production in animals fed S. cerevisiaebased additive [39-41], the biological significance of 
Table 1 Identified peak pairs (tier 1 and tier 2) that were affected by dietary supplementation of a blend of S. cerevisiaebased direct-fed microbials and fermentation products

\begin{tabular}{|c|c|c|}
\hline Compound & Fold change & FDR \\
\hline Galactose & 2.60 & $<0.01$ \\
\hline Lactose & 0.46 & $<0.01$ \\
\hline Glucose & 2.62 & $<0.01$ \\
\hline Fructose & 2.31 & $<0.01$ \\
\hline Isomer of fructose & 2.30 & $<0.01$ \\
\hline Isomer of glyceraldehyde & 2.01 & 0.01 \\
\hline Glyceraldehyde & 2.01 & 0.01 \\
\hline Hippuric acid & 2.13 & $<0.01$ \\
\hline Phenylacetylglycine & 1.98 & 0.01 \\
\hline 5-Hydroxykynurenamine & 2.63 & $<0.01$ \\
\hline 4-Oxoglutaramate & 1.82 & $<0.01$ \\
\hline 2-Dehydro-3-deoxy-D-glucarate & 1.80 & $<0.01$ \\
\hline 3-Fumarylpyruvate & 2.58 & $<0.01$ \\
\hline 1-Deoxy-D-xylulose 5-phosphate & 2.36 & $<0.01$ \\
\hline Glycolaldehyde & 1.63 & 0.01 \\
\hline Hydroxypyruvate & 1.60 & $<0.01$ \\
\hline 2-dehydro-3-deoxy-L-arabinonate & 0.30 & $<0.01$ \\
\hline Acetoacetate & 0.62 & 0.01 \\
\hline Dehydroascorbate - 2 tags & 1.74 & 0.01 \\
\hline 3-Methylindolepyruvate & 3.72 & $<0.01$ \\
\hline (S)-2-Aceto-2-hydroxybutanoate & 2.96 & $<0.01$ \\
\hline 5-Oxopentanoate & 2.30 & $<0.01$ \\
\hline (R)-3-Hydroxy-3-methyl-2-oxopentanoate & 2.96 & $<0.01$ \\
\hline 2-Dehydropantoate & 3.28 & $<0.01$ \\
\hline Isomer of (S)-2-aceto-2-hydroxybutanoate & 3.51 & $<0.01$ \\
\hline Isomer of (S)-3-methyl-2-oxopentanoic acid & 2.19 & $<0.01$ \\
\hline
\end{tabular}

FC: fold change relative to control

$P$-value was calculated from student's $t$-test

Tier 1 - Positive identification (chemical isotope labelling library);

Tier 2 - High confidence putative identification (linked identity library library)

Only metabolites with both fold-change $\geq 1.5$ or $\leq 0.67$ and FDR $\leq 0.01$

are shown increased plasma phenylacetylglycine is not known because ruminal ammonia level was not measured in this study. Further studies are needed to determine the relationship between blood phenylacetylglycine content and ruminal ammonia levels of beef cattle.

Limited or no information is available in the literature on the significance of the other metabolites affected by PROB supplementation in this study. Some of these metabolites such as 5-oxopentanoate, isomer of (S)-2-aceto2-hydroxybutanoate, isomer of (S)-3-methyl-2-oxopentanoic acid, 3-(4-hydroxyphenyl)pyruvate, (S)-2-aceto-2hydroxybutanoate, (R)-3-hydroxy-3-methyl-2-oxopentanoate, and 2-dehydropantoate were positively correlated with performance parameters such as ADG and FE. More in-depth studies are needed to understand better how these metabolites are associated with improved animal performance.

The primary limitation of this study was the use of an untargeted metabolomics approach which provides relative quantification of the metabolites based on intensity values. Nonetheless, this study confirms the benefits of PROB supplementation at improving the metabolic status of beef steers. Further studies that utilize a targeted metabolomics are needed to provide absolute quantification of some of the metabolites whose functions are currently unknown. Future advances in metabolomics technology should also focus on identifying those metabolites that were putatively matched in MCID library in this study.

\section{Effects of PROB on fecal bacterial community}

In the present study, the relative abundance of Prevotellaceae UCG-003 was increased in steers fed PROB diet and was the most altered taxa as revealed by LEfSe analysis. Prevotellaceae UCG-003 belongs to a group of bacteria, Prevotella. Prevotella plays a key role in the metabolisms of carbohydrate (such as sugar, starch and xylan) and protein [42-44]. Prevotella can grow effectively at low $\mathrm{pH}(<6.0)$, especially in the rumen $[45,46]$. Thus, the increased relative abundance of Prevotellaceae UCG-003 in the feces of beef steers fed PROB diet is

Table 2 Pearson correlations between plasma metabolites and performance indices of the beef steers

\begin{tabular}{|c|c|c|c|c|}
\hline & \multicolumn{2}{|l|}{ ADG } & \multicolumn{2}{|l|}{ FE } \\
\hline & r & $P$-value & r & $P$-value \\
\hline 3-(4-Hydroxyphenyl)pyruvate & 0.27 & 0.09 & 0.22 & 0.18 \\
\hline (S)-2-Aceto-2-hydroxybutanoate & 0.31 & 0.06 & 0.25 & 0.13 \\
\hline 5-Oxopentanoate & 0.43 & 0.01 & 0.36 & 0.03 \\
\hline (R)-3-Hydroxy-3-methyl-2-oxopentanoate & 0.31 & 0.06 & 0.24 & 0.14 \\
\hline 2-Dehydropantoate & 0.31 & 0.06 & 0.25 & 0.12 \\
\hline Isomer of (S)-2-aceto-2-hydroxybutanoate & 0.33 & 0.04 & 0.27 & 0.09 \\
\hline Isomer of (S)-3-methyl-2-oxopentanoic acid & 0.32 & 0.05 & 0.30 & 0.07 \\
\hline
\end{tabular}

Only metabolites with correlation coefficient ( $r$ ) of $P$-value $\leq 0.10$ for either average daily gain (ADG) or feed efficiency (FE) are shown 
Table 3 Relative abundance of the dominant fecal bacterial genera ( $>0.01 \%$ of total sequences) that were affected by dietary supplementation of a blend of S. cerevisiae-based directfed microbial and fermentation products

\begin{tabular}{lllll}
\hline Genus (\% of total sequences) & CON & PROB & SE & P-value \\
\hline Prevotellaceae UCG-003 & 1.91 & 4.15 & 0.48 & 0.03 \\
p-2534-18B5 gut group $^{\mathrm{a}}$ & 0.81 & 0.00 & 0.60 & 0.01 \\
Elusimicrobium $_{\text {Megasphaera }}$ & 0.26 & 0.01 & 0.18 & 0.02 \\
Moheibacter & 0.00 & 0.07 & 0.00 & 0.01 \\
Comamonas & 0.08 & 0.00 & 0.05 & 0.04 \\
Dorea & 0.06 & 0.00 & 0.04 & 0.01 \\
Stenotrophomonas & 0.07 & 0.15 & 0.01 & 0.02 \\
Blautia & 0.04 & 0.00 & 0.02 & 0.01 \\
Acetitomaculum & 0.04 & 0.09 & 0.01 & 0.01 \\
\hline
\end{tabular}

ancultured bacterium belonging to the indicated family

probably an increased production of organic acids in the hindgut due to increased fermentation of carbohydrates, which is associated with reduced fecal $\mathrm{pH}$ [47]. This probably explains the decreased fecal $\mathrm{pH}$ of the beef steers reported in our companion study [10]. These results suggest the viability of the DFM used in this study in the hindgut. Previous studies have confirmed the viability of $S$. cerevisiae and lactobacilli, both of which are constituents of $\mathrm{PROB}$, throughout the gastrointestinal tract of beef cattle [48, 49]. An alternative explanation for increased relative abundance of Prevotellaceae UCG-003 in the feces is that PROB supplementation reduced ruminal fermentation and caused a shift in the site of fermentation to the large intestine, thereby increasing substrate availability for increased growth of Prevotellaceae UCG-003. This scenario would be expected to reduce the performance of the animals, which is contradictory to the growth performance results reported in our companion paper [10], because the contribution of the hindgut fermentation to nutrient digestion in ruminants is substantially less than that of the rumen.

Supplementation of PROB increased the relative abundance of lactate-utilizing bacteria, such as Dorea and Megasphera. This suggests the activities of the lactate-producing bacterial content of PROB. Increased colonization of Lactobacillus in the hindgut could result in increased production of lactic acid, which can favor the growth of lactateutilizing bacteria. Blautia, a bacterial genus that can degrade complex carbohydrates, and Acetitomaculum, an

A.

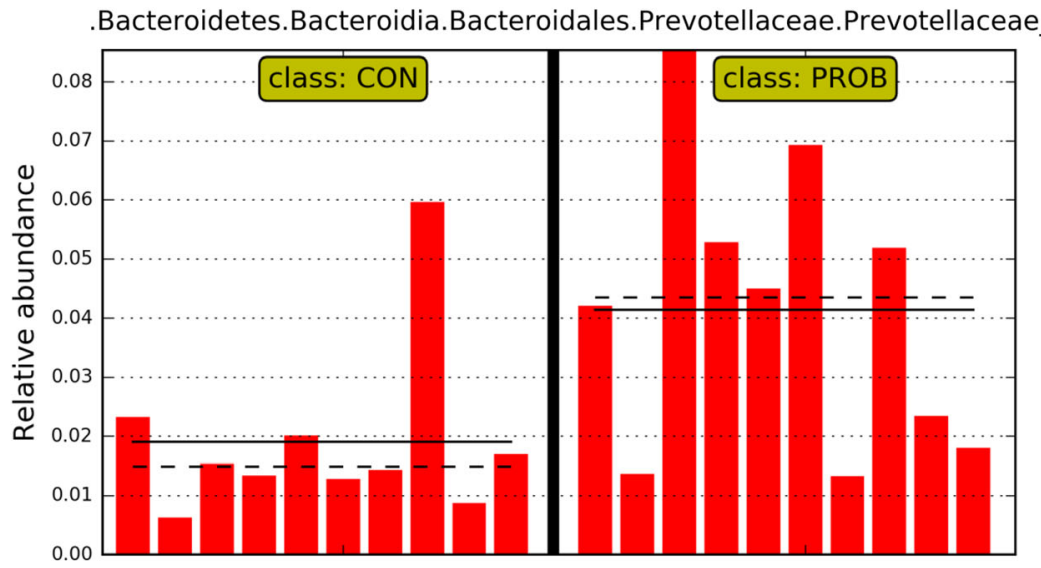

B.

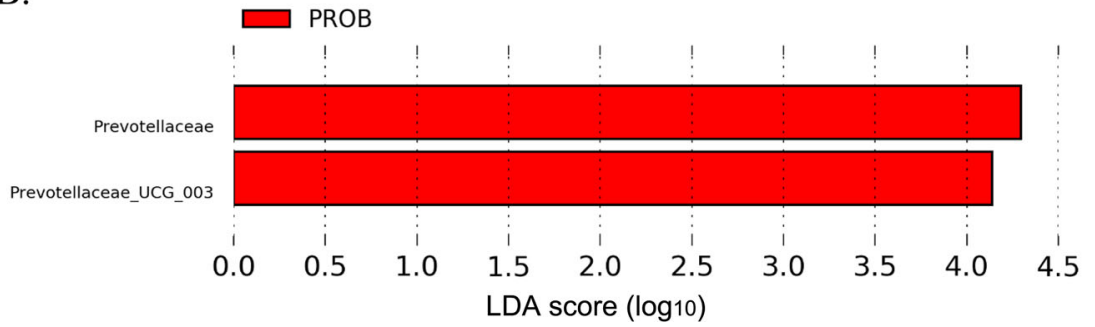

Fig. 3 a. Relative abundance of Prevotellaceae UCG-003 and b. Linear discriminant analysis effect size (LEfSe) of fecal bacterial population of beef steer fed no (CON) or $19 \mathrm{~g} / \mathrm{d}$ of a blend of S. cerevisiae-based direct-fed microbials and fermentation products (PROB; PMI, Arden Hills, MN, USA). Linear discriminant analysis effect size plot shows the most differentially abundant taxa according to the logarithmic linear discriminant analysis (LDA) $\geq 4.0$ 
acetogenic bacterial genus were also increased in feces of steers fed PROB. Conversely, the relative abundance of Elusimicrobium, Moheibacter, Stenotrophomonas, Comamonas, and uncultured bacterium belonging to family p-2534-18B5 gut group were reduced. The relative abundance of these aforementioned bacterial genera, including Dorea and Megasphera are low $(\leq 0.1 \%)$, therefore, changes in their relative abundance may not be of biological relevance. Taken together, these results indicate that PROB supplementation altered the fecal bacterial community toward increased relative abundance of Prevotellaceae UCG-003 and lactate-utilizing bacteria.

\section{Conclusion}

Supplementation of PROB improved the energy status of the beef steers by increasing the relative concentrations of plasma monosaccharides such as glucose, galactose, fructose, and glyceraldehyde, as well as others (hippuric acid, phenylacetylglycine, and 5-hydroxykynurenamine) with possible health benefits. In contrast, the relative concentration of acetoacetate was reduced. The significance of some metabolites, notably 5-oxopentanoate, isomer of (S)-2-aceto-2-hydroxybutanoate, isomer of (S)3-methyl-2-oxopentanoic acid, 3-(4-hydroxyphenyl)pyruvate, (S)-2-aceto-2-hydroxybutanoate, (R)-3-hydroxy-3methyl-2-oxopentanoate, and 2-dehydropantoate that showed positive correlations with performance measures merits further evaluation in future studies. Supplementation of PROB alters the fecal bacterial population towards increased relative abundance of Prevotellaceae UCG-003 and some.

\section{Supplementary information}

Supplementary information accompanies this paper at https://doi.org/10. 1186/s40104-019-0419-5.

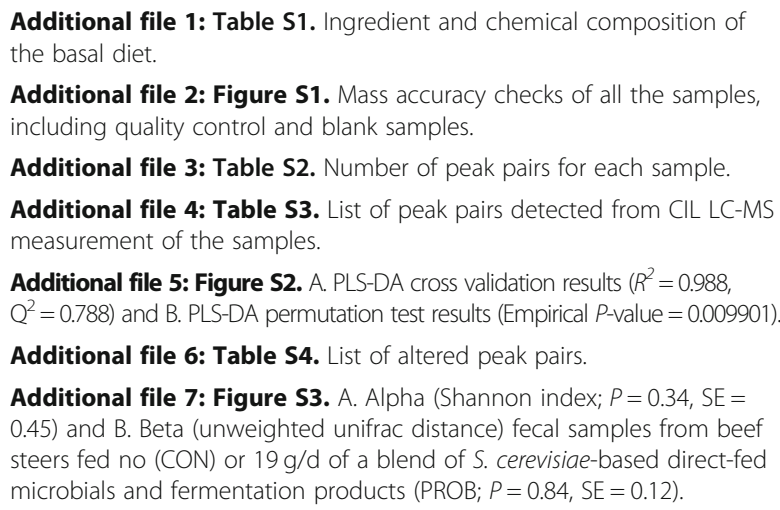

\section{Abbreviations}

ADG: Average daily gain; CIL: Chemical isotope labeling; DFM: Direct-fed microbial; DnsHz: Dansylhydrazine; FC: Fold change; FE: Feed efficiency; LCMS: Liquid chromatography mass spectrometry; LDA: Linear discriminant analysis; LEFSe: Linear discriminant analysis effect size; m/z: Mass-to-charge ratio; OTU: Operational taxonomic units; PCA: Principle component analysis;
PCR: Polymerase chain reaction; PLS-DA: Partial least squares discriminant analysis; PROB: A blend of Saccharomyces cerevisiae-based DFM and fermentation products; QC: Quality control; TMR: Total mixed ration

\section{Acknowledgements}

The authors are grateful to Hank Schweickart and Kyle Cannon for the daily care of the animals.

\section{Authors' contributions}

$\mathrm{IO}$ conceived and designed the experiment. $\mathrm{IO}$ and $\mathrm{JA}$ performed the experiment. IO, SOP, APC, and MDD performed the laboratory analysis. 10 wrote the manuscript. All authors read, critically revised for intellectual contents and approved the final manuscript.

\section{Funding}

The study was funded by PMI. Additional funding support was provided by the United States Department of Agriculture's National Institute of Food and Agriculture Evans-Allen project 1008985.

Availability of data and materials

All data generated or analyzed are available from the corresponding author on request.

\section{Ethics approval and consent to participate}

The research protocol (protocol number 18-001) was reviewed and approved by the Institutional Animal Care and Use Committee of Kentucky State University.

\section{Consent for publication}

Not applicable.

\section{Competing interests}

No conflict of interest declared.

\section{Author details}

${ }^{1}$ College of Agriculture, Communities, and the Environment, Kentucky State University, Frankfort, KY 40601, USA. ${ }^{2}$ Department of Animal Science, Berry College, Mount Berry, GA 30149, USA. ${ }^{3}$ Tecnologico de Monterrey, Escuela de Ingenieria y Ciencias, Queretaro, Mexico. ${ }^{4}$ Agricultural Research Station, Fort Valley State University, Fort Valley, GA 31030, USA.

Received: 30 September 2019 Accepted: 22 December 2019 Published online: 17 February 2020

\section{References}

1. Boyd J, West JW, Bernard JK. Effects of the addition of direct-fed microbials and glycerol to the diet of lactating dairy cows on milk yield and apparent efficiency of yield. J Dairy Sci. 2011;94:4616-22.

2. Broadway P, Carroll J, Sanchez N. Live yeast and yeast cell wall supplements enhance immune function and performance in food-producing livestock: a review. Microorganisms. 2015;3:417-27.

3. Xu H, Huang W, Hou Q, Kwok L, Sun Z, Ma H, et al. The effects of probiotics administration on the milk production, milk components and fecal bacteria microbiota of dairy cows. Sci Bull. 2017;62:767-74.

4. Sun P, Wang JQ, Deng LF. Effects of Bacillus subtilis natto on milk production, rumen fermentation and ruminal microbiome of dairy cows. Animal. 2013;7:216-22. https://doi.org/10.1017/S1751731112001188.

5. Qiao GH, Shan AS, Ma N, Ma QQ, Sun ZW. Effect of supplemental Bacillus cultures on rumen fermentation and milk yield in Chinese Holstein cows. J Anim Physiol Anim Nutr (Berl). 2010;94:429-36. https://doi.org/10.1111/j. 1439-0396.2009.00926.x.

6. Philippeau C, Lettat A, Martin C, Silberberg M, Morgavi DP, Ferlay A, et al. Effects of bacterial direct-fed microbials on ruminal characteristics, methane emission, and milk fatty acid composition in cows fed high- or low- starch diets. J Dairy Sci. 2017;100:2637-50.

7. Luan S, Duersteler M, Galbraith EA, Cardoso FC. Effects of direct-fed Bacillus pumilus 8G-134 on feed intake, milk yield, milk composition, feed conversion, and health condition of pre- and postpartum Holstein cows. J Dairy Sci. 2015; 98:6423-32.

8. Ghosson H, Schwarzenberg A, Jamois F, Yvin JC. Simultaneous untargeted and targeted metabolomics profiling of underivatized primary metabolites 
in sulfur-deficient barley by ultra-high performance liquid chromatographyquadrupole/time-of-flight mass spectrometry. Plant Methods. 2018;14:62.

9. Zhao S, Dawe M, Guo K, Li L. Development of high-performance chemical isotope labeling LC-MS for profiling the carbonyl submetabolome. Anal Chem. 2017:89:6758-65.

10. Adeyemi JA, Harmon DL, Paulus Compart DM, Ogunade IM. Effects of a blend of Saccharomyces cerevisiae-based direct-fed microbial and fermentation products in the diet of newly-weaned beef steers: growth performance, whole-blood immune gene expression, serum biochemistry and plasma metabolome. J Anim Sci. 2019;97:4657-67.

11. Fox JT, Depenbusch BE, Drouillard JS, Nagaraja TG. Dry-rolled or steamflaked grain-based diets and fecal shedding of Escherichia coli $\mathrm{O} 157$ in feedlot cattle. J Anim Sci. 2007:85:1207-12.

12. Magoc T, Salzberg SL. FLASH: fast length adjustment of short reads to improve genome assemblies. Bioinformatics. 2011;27:2957-63.

13. Bolger AM, Lohse M, Usadel B. Timmomatic: a flexible trimmer for illumina sequence data. Bioinformatics. 2014;30:2114-20. https://doi.org/10.1093/ bioinformatics/btu170.

14. Mukherjee S, Stamatis D, Bertsch J, Ovchinnikova G, Verezemska O, Isband $M$, et al. Genomes OnLine database (GOLD) v. 6: data updates and feature enhancements. Nucleic Acids Res. 2017:45:446-56.

15. Edgar RC, Haas BJ, Clemente JC, Quince C, Knight R. UCHIME improves sensitivity and speed of chimera detection. Bioinformatics. 2011;27:2194-200.

16. Caporaso JG, Kuczynski J, Stombaugh J, Bittinger K, Bushman FD, Costello EK, et al. QIIME allows analysis of high-throughput community sequencing data. Nat Methods. 2010;7:335-6.

17. Segata N, lzard J, Waldron L, Gevers D, Miropolsky L, Garrett WS, et al. Metagenomic biomarker discovery and explanation. Genome Biol. 2011;12:60

18. Mung D, Li L. Development of chemical isotope labeling LC-MS for milk metabolomics: comprehensive and quantitative profiling of the amine/ phenol submetabolome. Anal Chem. 2017;89:4435-43.

19. Huan T, Liang L. Quantitative metabolome analysis based on chromatographic peak reconstruction in chemical isotope labeling liquid chromatography mass spectrometry. Anal Chem. 2015;87:7011-6.

20. Li L, Li RH, Zhou JJ, Zuniga A, Stanislaus AE, Wu YM, et al. MyCompoundID: using an evidence-based metabolome library for metabolite identification. Anal Chem. 2013;85:3401-8.

21. Nafikov RA, Beitz DC. Carbohydrate and lipid metabolism in farm animals. J Nutr. 2007;137:702-5.

22. Aschenbach JR, Kristensen NB, Donkin SS, Hammon HM, Penner GB. Gluconeogenesis in dairy cows: the secret of making sweet milk from sour dough. IUBMB Life. 2010;62:869-677.

23. Nagaraja TG, Titgemeyer EC. Ruminal acidosis in beef cattle: the current microbiological and nutritional outlook. J Dairy Sci. 2007:90:17-38.

24. Martin SA, Nisbet DJ. Effect of direct-fed microbials on rumen microbial fermentation. J Dairy Sci. 1992;75:1736-44

25. Callaway ES, Martin SA. Effects of a Saccharomyces cerevisiae culture on ruminal bacteria that utilize lactate and digest cellulose. J Dairy Sci. 1997;80: 2035-44.

26. McAllister TA, Beauchemin KA, Alazzeh AY, Baah J, Teather RM, Stanford K. Review: the use of direct-fed microbials to mitigate pathogens and methane production in cattle. Can J Anim Sci. 2011;91:193-211.

27. Grummer RR. Etiology of lipid-related metabolic disorders in periparturient dairy cows. J Dairy Sci. 1993;76:3882-96.

28. Nocek JE, Kautz WP, Leedle JAZ, Block E. Direct fed microbial supplementation on the performance of dairy cattle during the transition period. J Dairy Sci. 2003:86:331-5.

29. Nocek JE, Kautz WP. Direct-fed microbial supplementation on ruminal digestion, health, and performance of pre- and postpartum dairy cattle. J Dairy Sci. 2006;89:260-6.

30. Lees HJ, Swann JR, Wilson ID, Nicholson JK, Holmes E. Hippurate: the natural history of a mammalian-microbial cometabolite. J Proteome Res. 2013;12: $1527-46$.

31. Williams RE, Eyton-Jones HW, Farnworth MJ, Gallagher R, Provan WM. Effect of intestinal microflora on the urinary metabolic profile of rats: a ${ }^{1} \mathrm{H}$-nuclear magnetic resonance spectroscopy study. Xenobiotica. 2002;32:783-94.

32. Nieman D, Kay C, Rathore A, Grace M, Strauch R, Stephan E, et al. Increased plasma levels of gut-derived phenolics linked to walking and running following two weeks of flavonoid supplementation. Nutrients. 2018;10:1718.

33. de Mello VD, Lankinen MA, Lindström J, Puupponen-Pimiä R, Laaksonen DE, Pihlajamäki J, et al. Fasting serum hippuric acid is elevated after bilberry
(Vaccinium myrtillus) consumption and associates with improvement of fasting glucose levels and insulin secretion in persons at high risk of developing type 2 diabetes. Mol Nutr Food Res. 2017;61:1700019. https:// doi.org/10.1002/mnfr.201700019.

34. Wu X, Sun H, Xue M, Wang D, Liu J. Serum metabolome profiling revealed potential biomarkers for milk protein yield in dairy cows. J Proteome. 2018;184:54-61.

35. Moffett JR, Namboodiri MA. Tryptophan and the immune response. Immunol Cell Biol. 2003;81:247-65.

36. Okuma M, Tokuyama T, Senoh S, Hirata F, Hayaishi O. Antagonism of 5hydroxykynurenamine against serotonin action on platelet aggregation. Proc Natl Acad Sci U S A. 1976;73(2):643-5.

37. Fujisaka S, Avila-Pacheco J, Soto M, Kostic A, Dreyfuss JM, Pan H, et al. Diet, genetics, and the gut microbiome drive dynamic changes in plasma metabolites. Cell Rep. 2018;22:3072-86.

38. Kristiansen $\mathrm{RG}$, Rose $\mathrm{CF}$, Fuskevåg $\mathrm{OM}$, Mæhre $\mathrm{H}$, Revhaug $\mathrm{A}$, Jalan $\mathrm{R}$, et al. L-ornithine phenylacetate reduces ammonia in pigs with acute liver failure through phenylacetylglycine formation: a novel ammonia-lowering pathway. Am J Physiol Gastrointest Liver Physiol. 2014;307:1024-31.

39. Chaucheyras-Durand F, Fonty G. Influence of a probiotic yeast (Saccharomyces cerevisiae CNCM I-1077) on microbial colonization and fermentations in the rumen of newborm lambs. Microb Ecol Health Dis. 2002;14:30-6.

40. Hristov AN, Varga G, Cassidy T, Long M, Heyler K, Karnati SKB, et al. Effect of Saccharomyces cerevisiae fermentation product on ruminal fermentation and nutrient utilization in dairy cows. J Dairy Sci. 2010;93:682-92.

41. Ogunade IM, Schweickart H, McCoun M, Cannon K, McManus C. Integrating 16S rRNA sequencing and LC-MS-based metabolomics to evaluate the effects of live yeast on rumen function in beef cattle. Animals. 2019;9:28.

42. Kabel MA, Yeoman CI, Han Y, Dodd D, Abbas CA, de Bont JA, et al. Biochemical characterization and relative expression levels of multiple carbohydrate esterases of the xylanolytic rumen bacterium Prevotella ruminicola 23 grown on an esterenriched substrate. Appl Environ Microbiol. 2011;77:5671-81.

43. Deusch S, Camarinha-Silva A, Conrad J, Beifuss U, Rodehutscord M, Seifert J. A structural and functional elucidation of the rumen microbiome influenced by various diets and microenvironments. Front Microbiol. 2017:8:1605.

44. Kim JN, Méndez-García C, Geier RR, lakiviak M, Chang J, Cann I, et al. Metabolic networks for nitrogen utilization in Prevotella ruminicola 23. Sci Rep. 2017;7:7851.

45. Russell JB, Dombrowski DB. Effect of pH on the efficiency of growth by pure cultures of rumen bacteria in continuous culture. Appl Environ Microbiol. 1980;39:604-10

46. Stevenson DM, Weimer PJ. Dominance of Prevotella and low abundance of classical ruminal bacterial species in the bovine rumen as revealed by relative quantification real-time PCR. Appl Microbiol Biotechnol. 2007;75:165-74.

47. Zinn RA, Barreras A, Corona L, Owens FN, Ware RA. Starch digestion by feedlot cattle: predictions from analysis of feed and fecal starch and nitrogen. J Anim Sci. 2007:85:1727-30

48. Fomenky BE, Chiquette J, Bissonnette N, Talbot G, Chouinard PY, IbeaghaAwemu EM. Impact of Saccharomyces cerevisiae boulardii CNCMI-1079 and Lactobacillus acidophilus BT1386 on total lactobacilli population in the gastrointestinal tract and colon histomorphology of Holstein dairy calves. Anim Feed Sci Technol. 2017;234:151-61.

49. Fomenky BE, Chiquette J, Lessard M, Bissonnette N, Talbot G, Chouinard YP, et al. Saccharomyces cerevisiae var. boulardii CNCM I-1079 and Lactobacillus acidophilus BT1386 influence innate immune response and serum levels of acute-phase proteins during weaning in Holstein calves. Can J Anim Sci. 2018;98:576-88.

\section{Ready to submit your research? Choose BMC and benefit from:}

- fast, convenient online submission

- thorough peer review by experienced researchers in your field

- rapid publication on acceptance

- support for research data, including large and complex data types

- gold Open Access which fosters wider collaboration and increased citations

- maximum visibility for your research: over $100 \mathrm{M}$ website views per year

At BMC, research is always in progress.

Learn more biomedcentral.com/submissions 